\title{
Antibacterial Activity of Dental Cements Containing Quaternary Ammonium Polyethylenimine Nanoparticles
}

\author{
Nurit Beyth, ${ }^{1}$ Raphael Pilo, ${ }^{2}$ and Ervin I. Weiss ${ }^{1}$ \\ ${ }^{1}$ Department of Prosthodontics, Faculty of Dental Medicine, Hebrew University-Hadassah School of Dental Medicine, \\ P.O. Box 12272, Jerusalem 91120, Israel \\ ${ }^{2}$ Department of Prosthetic Dentistry, Goldschleger School of Dental Medicine, Tel-Aviv University, Tel Aviv 69978, Israel
}

Correspondence should be addressed to Nurit Beyth, nuritb@ekmd.huji.ac.il

Received 25 January 2012; Revised 19 March 2012; Accepted 20 March 2012

Academic Editor: Zhongkui Hong

Copyright (C) 2012 Nurit Beyth et al. This is an open access article distributed under the Creative Commons Attribution License, which permits unrestricted use, distribution, and reproduction in any medium, provided the original work is properly cited.

\begin{abstract}
Glass ionomer cements (GICs) are commonly used for cementing full cast crown restorations. Regrettably, although the dental cements fill the gap between the tooth and the crown, bacterial microleakage may occur, resulting in secondary caries. As microleakage cannot be completely prevented, GICs possessing antibacterial properties are in demand. In the present study the antibacterial activity of insoluble, cross-linked quaternary ammonium polyethylenimine (QPEI) nanoparticles incorporated at $1 \% \mathrm{w} / \mathrm{w}$ in two clinically available GICs were studied. The antibacterial activity was tested against Streptococcus mutans and Lactobacillus casei using the direct contact test (DCT) and the agar diffusion test (ADT). Using the direct contact test, antibacterial activity $(P<0.05)$ was found in both tested GICs with incorporated QPEI nanoparticles, the effect lasting for at least one month. However, the ADT showed no inhibition halo in the test bacteria, indicating that the antimicrobial nanoparticles do not diffuse into the agar. The results show that the incorporation of QPEI nanoparticles in glass ionomer cements has a long-lasting antibacterial effect against Streptococcus mutans and Lactobacillus casei. Changing the antibacterial properties of glass ionomer cements by incorporating QPEI antibacterial nanoparticles may prolong the clinical performance of dental crowns.
\end{abstract}

\section{Introduction}

Most of the currently used dental restorative materials do not form a long-lasting perfect seal with the cavity wall. Indeed, it has been shown that gap formation and microleakage are not completely prevented, even when using state-of-the-art bonding systems [1]. Gaps between restoration margins and cavity walls are colonized by oral microorganisms and may result in secondary caries formation and pulp disease $[2,3]$. Secondary caries has been identified as the major factor responsible for the longevity of dental restorations [4-6].

Traditionally, cements, such as glass-ionomer cements, are used to fill gaps and serve as sealing agents between the tooth preparation and dental crowns. Unfortunately, although cements are intended to seal the crown margins, bacterial microleakage may occur, resulting in secondary caries and thus compromising the longevity of the restoration [7]. Nonetheless, several in vitro studies have confirmed that GICs have an antibacterial potential [8-12]. These antibacterial properties are attributed to fluoride release, which is believed to inhibit microbial growth and metabolism. However, the fluoride is released within a short interval and the antibacterial effect is lost over time [13]. Accordingly, development of new GICs with distinct antibacterial properties is essential and may increase the longevity of dental crowns. Moreover, therapeutic benefits may be gained upon combining antibacterial agents with GIC materials.

As the growth-inhibitory effects of cements and bonding systems prevent bacterial colonization in marginal gaps, attempts have been made to decrease the bacterial counts under the restorations by incorporating leachable antimicrobial agents in the restorative materials [14-16]. Nevertheless, modification of dental materials with nonleachable antibacterial agents may be advantageous when preparing materials that undergo continuous physical challenged in the harsh oral environment for many years. Consequently a different approach based on the use of polymers with antibacterial properties, such as polycationic-type polymers [17, 18]. It is generally accepted that cationic polymers was developed with quaternary ammonium groups can be used 
as potent antimicrobials; bacteria do not appear to develop resistance to cationic polymers due to their nonspecific mode of action [19]. Several reports have described that following the incorporation of a methacryloyloxydodecyl pyridinium bromide (MDPB) monomer in resin composites, there was no release of the incorporated monomer and the resin composite exhibited antibacterial properties [20,21]. Previously, we showed that incorporation of small amounts of quaternized polyethyleneimine (QPEI) nanoparticles into resin composites exerts a strong antibacterial effect against a wide range of bacteria for at least one month, with no measurable effect on biocompatibility [22]. Additionally, we found that small amounts of QPEI nanoparticles can be immobilized in resin composites during polymerization without leaching out and without compromising the mechanical properties of the material [18]. Furthermore, we observed that QPEI nanoparticle quaternization with octyl groups yields optimal antibacterial properties for dental composite restorative material: six months aging of composite incorporating $1 \% \mathrm{w} / \mathrm{w}$ QPEI nanoparticles resulted in complete inhibition of cariogenic mutans streptococci biofilm in vitro [23]. More recently, the biocidal effect of incorporated antibacterial nanoparticles on in vivo oral biofilm was shown [23]. In the present study, we further investigated the effect of QPEI nanoparticles incorporated in two conventional GICs on Streptococcus mutans and Lactobacillus casei.

\section{Materials and Methods}

2.1. QPEI Nanoparticle Preparation. The synthesis of quaternary ammonium PEI nanoparticle was previously described by Beyth et al. [18, 22]. Briefly, PEI (10 g, 0.23 mol monomer units) dissolved in $100 \mathrm{~mL}$ ethanol was reacted with dibromopentane at a 1:0.04 mol ratio (monomer units of PEI/ dibromopentane) under reflux for $24 \mathrm{hrs}$. $\mathrm{N}$-alkylation was conducted as follows: octyl halide was added at a 1:1 mol ratio (monomer units PEI/octyl halide). Alkylation was carried out under reflux for $24 \mathrm{hrs}$, followed by neutralization with sodium hydroxide ( 1.25 equimolar, $0.065 \mathrm{~mol}$ ) for an additional $24 \mathrm{hrs}$ under the same conditions. $\mathrm{N}$-methylation was conducted as follows: $43 \mathrm{~mL}(0.68 \mathrm{~mol})$ of methyl iodide was added and methylation was continued at $42^{\circ} \mathrm{C}$ for $48 \mathrm{hrs}$ followed by neutralization with sodium bicarbonate $(0.23 \mathrm{~mol}, 19 \mathrm{~g})$ for an additional $24 \mathrm{hrs}$. The supernatant obtained was decanted and precipitated in $300 \mathrm{~mL}$ of doubledistilled water (DDW), washed with hexane and DDW, and then freeze-dried. The purification step was repeated using additional amounts of hexane and DDW. The average yield was 70\% (mol/mol). FT-IR (QPEI nanoparticles, $\mathrm{KBr}$ ): $3,440 \mathrm{~cm}^{-1}(\mathrm{~N}-\mathrm{H}), 2,956,2,926$, and $2,853 \mathrm{~cm}^{-1}(\mathrm{C}-\mathrm{H})$, $1,617 \mathrm{~cm}^{-1}\left(\mathrm{~N}-\mathrm{H}\right.$, small band), $1,465 \mathrm{~cm}^{-1}(\mathrm{C}-\mathrm{H}), 967 \mathrm{~cm}^{-1}$ quaternary nitrogen. $1 \mathrm{H}-\mathrm{NMR}$ (DMSO): $0.845 \mathrm{ppm}(\mathrm{t}, 3 \mathrm{H}$, $\mathrm{CH} 3$, octane hydrogens), $1.24 \mathrm{ppm}(\mathrm{m}, 10 \mathrm{H},-\mathrm{CH} 2-$, octyl hydrogens), $1.65 \mathrm{ppm}(\mathrm{m}, 2 \mathrm{H}, \mathrm{CH}$, octyl hydrogens), and $3.2-3.6 \mathrm{ppm}(\mathrm{m}, \mathrm{CH} 3$ of quaternary amine, $4 \mathrm{H},-\mathrm{CH} 2-$, PEI hydrogens, and $2 \mathrm{H},-\mathrm{CH} 2-$, octyl hydrogens.)

2.2. Preparation of Bacterial Suspension. The cariogenic bacteria Streptococcus mutans (ATCC 700610) and Lactobacillus casei (ATCC 334) were cultured overnight in $5 \mathrm{~mL}$ of brainheart infusion (BHI) (Difco, Detroit, MI, USA) broth, at $37^{\circ} \mathrm{C}$. To prevent the formation of large bacterial aggregates or long streptococcal chains, the top $4 \mathrm{~mL}$ of the undisturbed bacterial culture was transferred to a fresh test tube and centrifuged for $10 \mathrm{~min}$ at $3175 \times \mathrm{g}$. The supernatant was discarded, and the bacteria were resuspended in $5 \mathrm{~mL}$ of phosphate-buffered-saline (PBS) (Sigma, St. Louis, MO, USA) and vortexed gently for $10 \mathrm{sec}$. Each bacterial suspension was adjusted to an optical density of 1.0 at $650 \mathrm{~nm}$. Volumes of $10 \mu \mathrm{L}$ from tenfold serial dilutions were plated on $\mathrm{BHI}$ agar to determine colony-forming units/mL.

2.3. Preparation of Test Samples. The experimental specimens were prepared by adding the synthesized nanoparticles to two commercial glass ionomer cements (GICs): GC Fuji I (GC, Tokyo, Japan) and Ketac-Cem (3 M ESPE, St. Paul, MN, USA). Nanoparticle powder was added $(1 \% \mathrm{w} / \mathrm{w})$ to the GICs and homogeneously mixed, according to the manufacturer's instructions.

2.4. Preparation of Microtiter Plates. Two clinically available GIC materials, Fuji I and Ketac-Cem, with the incorporated $1 \% \mathrm{w} / \mathrm{w}$ synthesized QPEI nanoparticles were tested. A microtiter plate (96-well flat bottom plate Nunclon, Nunc, Copenhagen, Denmark) was vertically positioned. Using a flat-ended dental instrument (dental spatula), the sidewalls of 8 wells were coated evenly with an equal amount of the same material tested $(25 \pm 5 \mathrm{mg}$ in each well). Special care was taken to leave the bottom of the well untouched in order to avoid false readings during incubation in the spectrophotometer. The materials were left to set according to the manufacturer's instructions. Additional sets of wells in the same microtiter plate coated with the original composite resins without QPEI particles served as control.

2.5. Material Aging. The microtiter plates were aged for one week and one month. During this time each well was filled with $250 \mu \mathrm{L}$ PBS, which was replaced every $48 \mathrm{hrs}$, and the plates were incubated at $37^{\circ} \mathrm{C}$. Before the direct contact tests, the PBS was aspirated and the plates were dried under sterile conditions.

2.6. Direct Contact Test. A $10 \mu \mathrm{L}$ volume of bacterial suspension $\left(\sim 10^{9}\right.$ bacteria) was placed on the surface of each tested material in a set of 8 wells, and the plate was incubated in a vertical position for $1 \mathrm{hr}$ at $37^{\circ} \mathrm{C}$. During the incubation period, the suspension liquid evaporated and a thin layer of bacteria was obtained, ensuring direct contact between all the bacteria and the tested surface. The plate was then positioned horizontally, and $220 \mu \mathrm{L}$ of BHI broth were added to each well containing the material.

To allow interexperimental comparison as described previously by Beyth et al. [24], each microtiter plate also included a set of wells for calibration of bacterial outgrowth. A $10 \mu \mathrm{L}$ volume of the bacterial suspension was placed on the sidewalls of two uncoated wells; $275 \mu \mathrm{L}$ of BHI broth was added and the plates were gently mixed for $30 \mathrm{sec}$. A $55 \mu \mathrm{L}$ sample from each well was transferred to an adjacent set of 


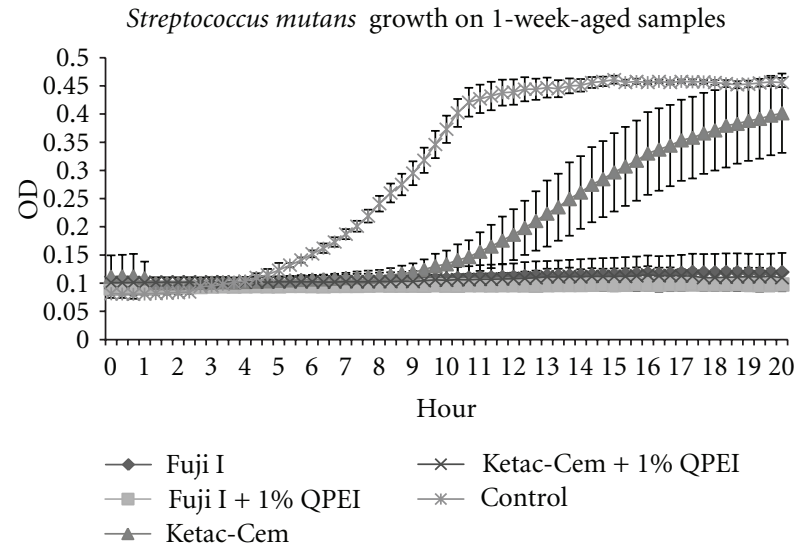

(a)

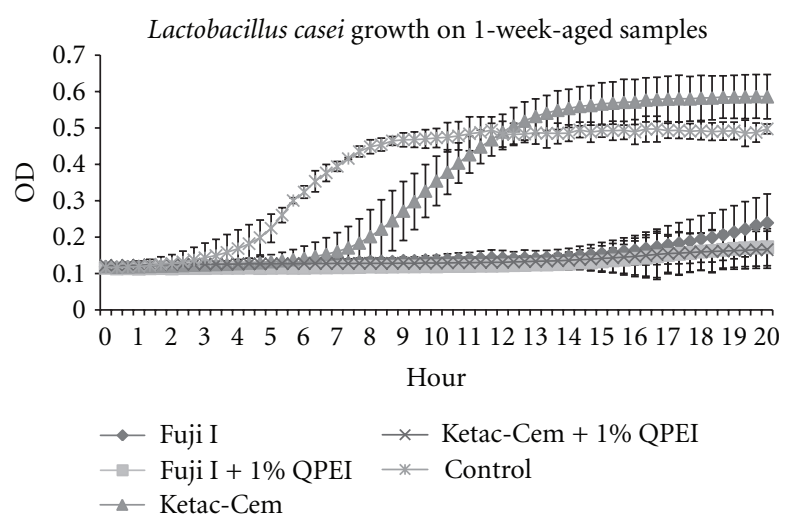

(b)

FIGURE 1: Streptococcus mutans (a) and Lactobacillus casei (b) growth measured following direct contact with one-week-aged glass ionomer cements (Fuji I and Ketac-Cem) incorporating 0 and 1\% w/w QPEI nanoparticles. Growth of the shed bacteria from the biofilm was measured every $20 \mathrm{~min}$ for $20 \mathrm{hrs}$. Each point on the curve is the average $( \pm \mathrm{SD})$ optical density simultaneously recorded in eight wells similarly prepared in the same microtiter plate. Each experiment was repeated 3 times.

wells containing $220 \mu \mathrm{L}$ of medium and the fivefold dilution was repeated six consecutive times. A gradual and reproducible decrease in optical density correlated with serial dilution. This also indicates that the initial number of viable bacteria had no effect on growth rate or on the final optical density in the stationary phase.

2.7. Kinetic Measurement of Bacterial Growth. The microtiter plate was placed in a temperature-controlled microplate spectrophotometer at $37^{\circ} \mathrm{C}$ (VERSAmax, Molecular Devices Corporation, Menlo Oaks Corporate Centre, Menlo Park, CA, USA), with $5 \mathrm{sec}$ vortex mixing prior to each reading. Bacterial outgrowth was estimated by following changes in optical density (OD) in each well at $650 \mathrm{~nm}$ every 20 minutes for $20 \mathrm{hrs}$.

2.8. Data Analysis. The optical density measurements were plotted, providing bacterial growth curves for each well in the microtiter plate. The linear portion of the logarithmic growth phase was subjected to statistical analysis. The results are expressed according to two variables: the slope $(a)$ and the constant $(b)$ of the linear function $a x+b=y$ derived from the ascending portion of the bacterial growth curve. The slope $(a)$ and the constant $(b)$ correlate with growth rate and initial bacterial number, respectively. The data were analyzed by one-way ANOVA and the Tukey multiple comparison test. The level of significance was determined as $P<0.05$.

2.9. Agar Diffusion Test. A $200 \mu \mathrm{L}$ volume of the S. mutans suspension was spread on Mitis Salivarius agar (MSB) (Difco, Detroit, MI) supplemented with bacitracin $0.0625 \mathrm{gr} / \mathrm{mL}$ (Sigma), and cured disc specimens of each tested material (clinically available GIC, and GIC incorporating 1\% w/w QPEI nanoparticles) were placed on the surface. The plates were incubated for $48 \mathrm{hrs}$ at $37^{\circ} \mathrm{C}$, and the inhibition zone around each specimen was measured. Blood agar plates were similarly processed for $L$. casei.

\section{Results}

3.1. Direct Contact Test. The average optical density measurements of $S$. mutans growth in 8 wells after direct contact with the one-week-aged materials are shown in Figure 1(a), comparing the antibacterial effect of the two clinically available GIC materials with and without the added QPEI nanoparticles. In the samples incorporating QPEI, no bacterial growth was evident. Additionally, it can be seen that the clinically available Fuji I totally inhibited bacterial growth whereas Ketac-Cem had a minor antibacterial effect. Similar results were obtained for L. casei (Figure 1(b)). Figures 2(a) and 2(b) depict the same experiment conducted after an aging process of one month. The antibacterial effect of the GICs with the incorporated synthesized QPEI lasted for at least one month, whereas both Fuji I and Ketac-Cem showed a limited antibacterial effect in aged samples. Analysis of the data obtained from the two tested microorganisms is shown in Figure 3. The data are expressed as the slope of the regression line plotted from the logarithmic growth phase of each bacterium. Slope values correlating with the growth rate are expressed as the percentage of the controls, normalized to $100 \%$. Data analysis of the absorbance measurements showed total growth inhibition $(P<0.05)$ in all samples with incorporated QPEI nanoparticles, regardless of the aging time of the tested materials.

3.2. Agar Diffusion Test. The clinically available GICs and the GICs with incorporated QPEI did not yield an inhibition zone on the agar plates inoculated with S. mutans and L. casei (Figures 4(a) and 4(b), resp.).

\section{Discussion}

The ability to reduce bacterial growth would lower the risk of further demineralization and cavitation, since dental caries is an infectious disease and eradication of cariogenic bacteria is 


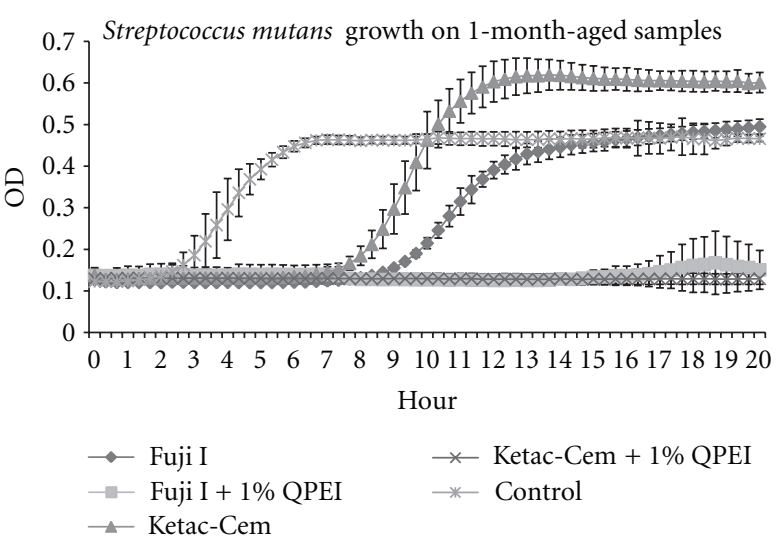

(a)

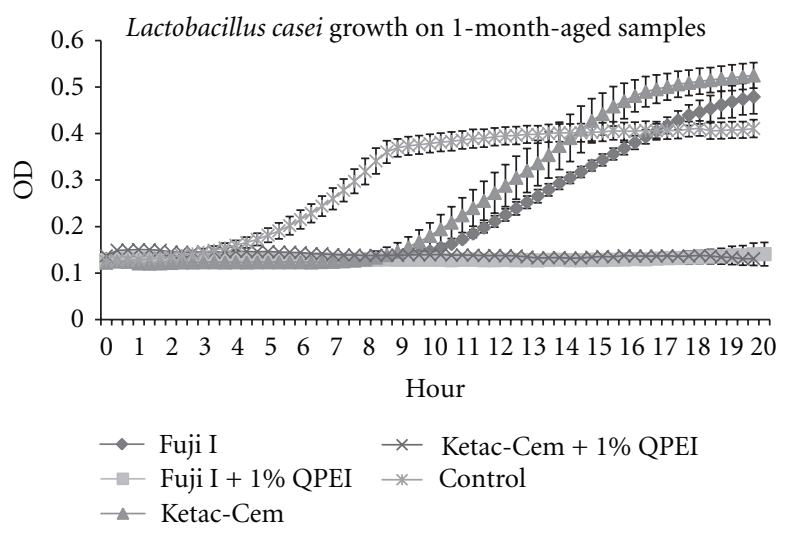

(b)

FIGURE 2: Streptococcus mutans (a) and Lactobacillus casei (b) growth measured following direct contact with one-month-aged glass ionomer cements (Fuji I and Ketac-Cem) incorporating 0 and 1\% w/w QPEI nanoparticles. Growth of the shed bacteria from the biofilm was measured every $20 \mathrm{~min}$ for $20 \mathrm{hrs}$. Each point on the curve is the average $( \pm \mathrm{SD})$ optical density simultaneously recorded in eight wells similarly prepared in the same microtiter plate. Each experiment was repeated 3 times.

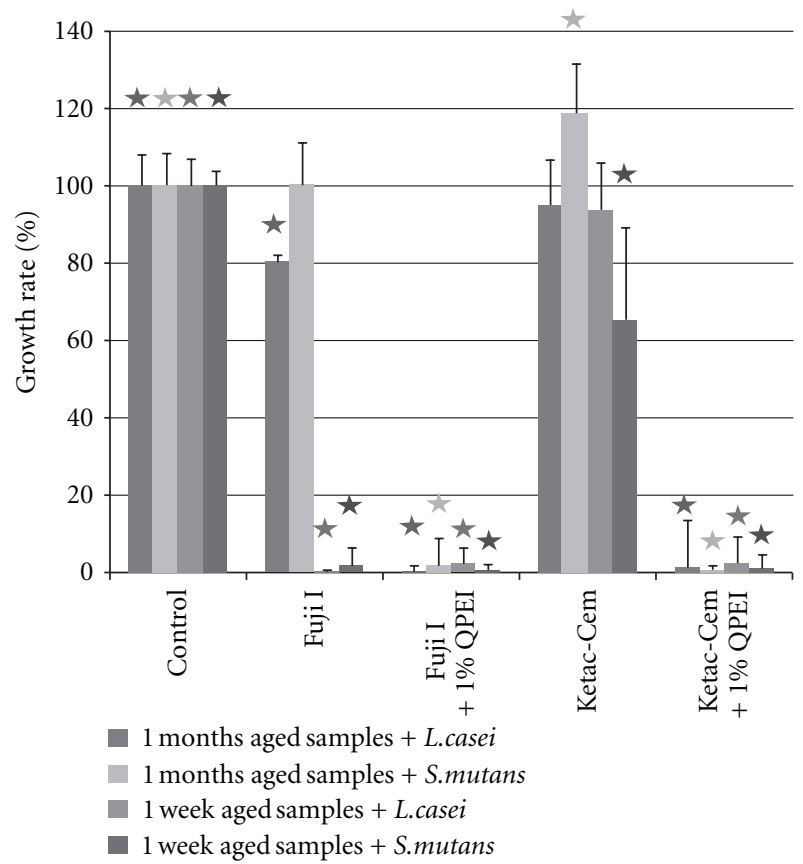

FIgURE 3: Bacterial growth rate (percentage) of Streptococcus mutans and Lactobacillus casei following direct contact with aged glass ionomer cements (Fuji I and Ketac-Cem) incorporating 0 and $1 \% \mathrm{w} / \mathrm{w}$ QPEI nanoparticles. The growth rate was determined in separate experiments in which samples were aged for one week or one month. The results are expressed as the mean $\pm \mathrm{SD}$; significant differences between the appropriate control and the different test groups are indicated by an asterisk $(P<0.05)$.

the guiding therapeutic principle. In the present study the antibacterial effect of clinically available GICs was achieved by incorporating quaternary ammonium polyethylenimine insoluble nanoparticles. The in vitro results showed that these cationic polymeric nanoparticles when incorporated at a low concentration (1\%) into the cements exerted a strong antibacterial activity against the tested bacteria regardless of the cement to which they were added. Total bacterial growth inhibition $(P<0.05)$ was also recorded in the one-week-aged samples of Fuji I. This effect diminished with the aging process. Interestingly, there was measurable antibacterial activity $(P<0.05)$ of both GICs tested in the one month-aged samples and in the Ketac-Cem one-week-aged samples. In these samples the antibacterial effect was mainly seen as a significant horizontal shift of the growth curve, whereas the growth rate of the bacteria was decreased only to some extent. The shift can be attributed to the considerably increased lag time of the cells by more than $40 \%$, as reflected by the slower transition of the cultures into the log phase. In these test samples, the bacterial cells reached the target induction point about $4.5 \mathrm{hrs}$ after the control bacteria. Thus, it may be speculated that growth in these cultures was initiated more slowly. Another possibility deducted from the calibration curves used in the present study to estimate the bacterial population is that the horizontal shift correlated with the calibration growth curve of diluted bacteria from the original tube at a fivefold dilution. Thus, an additional explanation might be that the bacterial population was reduced to less than $40 \%$ following contact with the surface of the tested materials, compared with the control. This coincides with other studies showing the antibacterial properties of GICs [25-27].

Most studies have investigated the antibacterial effect of freshly mixed GICs, whereas, in the present study, aged materials were used. Aged dental materials are known to have less antibacterial activity than freshly mixed specimens $[18,28]$, the reason being that some of the antibacterial components are washed away in the aging process or set into the material bulk. Our findings are in agreement with those of Yap et al. [12] who also reported that conventional GICs when set had no antibacterial effect in an agar diffusion test.

Different in vitro methods have been used in the past to study the antimicrobial activity of dental materials with the 


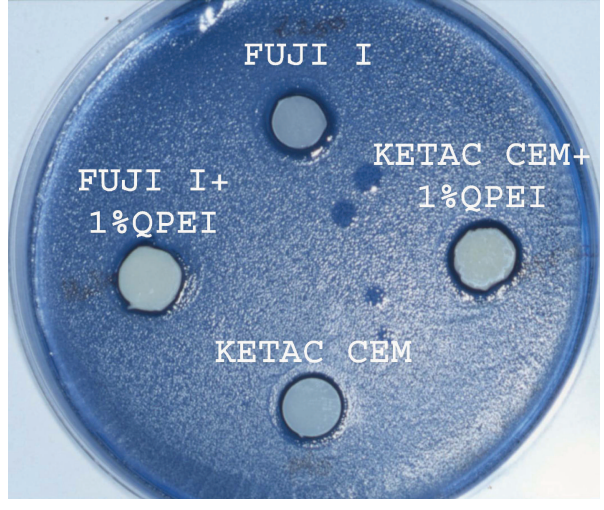

(a)

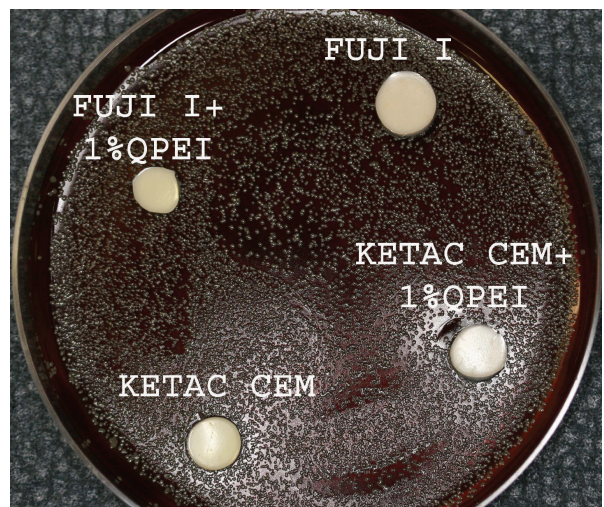

(b)

FIGURE 4: Results of agar diffusion test using glass ionomer cements (Fuji I and Ketac-Cem) incorporating 0 and 1\% w/w QPEI nanoparticles and Streptococcus mutans and Lactobacillus casei (a) and (b), respectively.

aim of inhibiting secondary caries at the restoration margin. Most methods used for this purpose, for example, traditional agar diffusion, do not adequately reflect clinical conditions. Therefore, in this study we used the DCT to determine the bacterial growth after incubation of a small amount of bacterial suspension on the materials surface. We also resorted to the agar diffusion test (ADT) as a tool for comparison with the existing published data. The ADT is a semiquantitative test originally used for evaluating the antibacterial properties of antibiotics. Although the ADT is widely used in the dental literature, it is unsuitable for testing materials which are designated to be insoluble, such as various restorative materials. Alternatively, the DCT was designed to evaluate quantitatively the antibacterial surface properties of materials with low to extremely low solubility. In this test, the bacteria are allowed to come in direct contact, under controlled conditions, with the tested material, to study the kinetics of bacterial growth [24].

The QPEI nanoparticles were previously reported to exert antibacterial properties when immobilized in resin composites, with no recorded effect of eluted particles [18, 22]. In the present study these insoluble antibacterial nanoparticles were found to be active for at least one month after being in contact with the tested bacteria. The bioactive components did not affect bacterial growth in the ADT. Accordingly, it may be speculated that the antibacterial activity is not through released particles.

The bacterial strains used throughout the experiments were S. mutans and L. casei, two microorganisms that play a major role in dental biofilm formation and in the etiology of caries. Regrettably, the tests used in the present study cannot fully simulate the complex biofilm formed in the oral cavity. Thus, further in vivo investigations are needed to test the antibacterial efficacy of the added QPEI nanoparticles.

The activity of polycationic agents such as polyethyleneimine is presumed to be through adsorption of the positively charged polymers onto the negatively charged bacterial cell surface resulting in increased cell permeability and disruption of the cell membranes [19]. This mode of action may have caused total inhibition of bacterial growth after direct contact with the samples incorporating QPEI nanoparticles. Additionally, the antibacterial effect could have resulted from the release of fluoride from the GICs [12, 29, 30]. However, this effect was found to be reduced in the clinically available materials during the aging process, whereas, in the materials incorporating QPEI, strong antibacterial inhibition was maintained.

In conclusion, the present study using antibacterial tests with $S$. mutants and L. casei shows that QPEI nanoparticles when incorporated in GICs at a low concentration (1\%) exhibit a strong antibacterial effect which lasts for at least one month. Incorporation of antibacterial nanoparticles in GICs may prevent biofilm formation and secondary caries.

\section{Conflict of Interests}

A patent entitled "Antimicrobial Nanoparticulate Additives Forming Non-Leachable Sustained Antimicrobial Polymeric Compositions" is pending approval.

\section{Acknowledgments}

The authors would like to acknowledge the Israeli Branch of GC EUROPE and the Israel Branch of $3 \mathrm{M}$ ESPE for kindly supplying the commercial glass ionomer cements used in the study.

\section{References}

[1] B. Haller and A. Trojanski, "Effect of multi-step dentin bonding systems and resin-modified glass ionomer cement liner on marginal quality of dentin-bonded resin composite Class II restorations," Clinical Oral Investigations, vol. 2, no. 3, pp. 130-136, 1998.

[2] C. González-Cabezas, Y. Li, T. W. Noblitt, R. L. Gregory, A. H. Kafrawy, and G. K. Stookey, "Detection of mutans streptococci in secondary carious lesions using immunofluorescent techniques and confocal laser scanning microscopy," Caries Research, vol. 29, no. 3, pp. 198-203, 1995.

[3] E. A. Kidd, F. Toffenetti, and I. A. Mjör, "Secondary caries," International Dental Journal, vol. 42, no. 3, pp. 127-138, 1992. 
[4] I. A. Mjör, C. Shen, S. T. Eliasson, and S. Richter, "Placement and replacement of restorations in general dental practice in Iceland," Operative Dentistry, vol. 27, no. 2, pp. 117-123, 2002.

[5] J. N. Walton, F. M. Gardner, and J. R. Agar, "A survey of crown and fixed partial denture failures: length of service and reasons for replacement," The Journal of Prosthetic Dentistry, vol. 56, no. 4, pp. 416-421, 1986.

[6] I. A. Mjör, "The reasons for replacement and the age of failed restorations in general dental practice," Acta Odontologica Scandinavica, vol. 55, no. 1, pp. 58-63, 1997.

[7] W. E. Van Amerongen and T. Pilot, "Dental caries under glass ionomer restorations," Journal of Public Health Dentistry, vol. 56, no. 3, pp. 150-154, 1996.

[8] Y. Benderli, H. Ulukapi, O. Balkanli, and G. Külekçi, "In vitro plaque formation on some dental filling materials," Journal of Oral Rehabilitation, vol. 24, no. 1, pp. 80-83, 1997.

[9] K. H. Friedl, G. Schmalz, K. A. Hiller, and M. Shams, "Resin-modified glass ionomer cements: fluoride release and influence on Streptococcus mutans growth," European Journal of Oral Sciences, vol. 105, no. 1, pp. 81-85, 1997.

[10] M. Herrera, A. Castillo, P. Baca, and P. Carrión, "Antibacterial activity of glass-ionomer restorative cements exposed to cavity-producing microorganisms," Operative Dentistry, vol. 24, no. 5, pp. 286-291, 1999.

[11] J. C. Meiers and G. A. Miller, "Antibacterial activity of dentin bonding systems, resin-modified glass ionomers, and polyacid-modified composite resins," Operative Dentistry, vol. 21, no. 6, pp. 257-264, 1996.

[12] A. U. Yap, E. Khor, and S. H. Foo, "Fluoride release and antibacterial properties of new-generation tooth-colored restoratives," Operative Dentistry, vol. 24, no. 5, pp. 297-305, 1999.

[13] I. Lewinstein, S. Matalon, S. Slutzkey, and E. I. Weiss, "Antibacterial properties of aged dental cements evaluated by direct-contact and agar diffusion tests," Journal of Prosthetic Dentistry, vol. 93, no. 4, pp. 364-371, 2005.

[14] N. Hiraishi, C. K. Yiu, N. M. King, and F. R. Tay, "Effect of chlorhexidine incorporation into a self-etching primer on dentine bond strength of a luting cement," Journal of dentistry, vol. 38, no. 6, pp. 496-502, 2010.

[15] A. Hoszek and D. Ericson, "In vitro fluoride release and the antibacterial effect of glass lonomers containing chlorhexidine gluconate," Operative Dentistry, vol. 33, no. 6, pp. 696-701, 2008.

[16] I. Lewinstein, H. Chweidan, S. Matalon, and R. Pilo, "Retention and marginal leakage of provisional crowns cemented with provisional cements enriched with chlorhexidine diacetate," Journal of Prosthetic Dentistry, vol. 98, no. 5, pp. 373378, 2007.

[17] S. Imazato, F. R. Tay, A. V. Kaneshiro, Y. Takahashi, and S. Ebisu, "An in vivo evaluation of bonding ability of comprehensive antibacterial adhesive system incorporating MDPB," Dental Materials, vol. 23, no. 2, pp. 170-176, 2007.

[18] N. Beyth, I. Yudovin-Farber, R. Bahir, A. J. Domb, and E. I. Weiss, "Antibacterial activity of dental composites containing quaternary ammonium polyethylenimine nanoparticles against Streptococcus mutans," Biomaterials, vol. 27, no. 21, pp. 3995-4002, 2006.

[19] B. Gao, X. Zhang, and Y. Zhu, "Studies on the preparation and antibacterial properties of quaternized polyethyleneimine," Journal of Biomaterials Science, Polymer Edition, vol. 18, no. 5, pp. 531-544, 2007.

[20] S. Imazato, T. Imai, R. R. Russell, M. Torii, and S. Ebisu, "Antibacterial activity of cured dental resin incorporating the antibacterial monomer MDPB and an adhesion-promoting monomer," Journal of Biomedical Materials Research, vol. 39, no. 4, pp. 511-515, 1998.

[21] S. Imazato, Y. Kinomoto, H. Tarumi, M. Torii, R. R. B. Russell, and J. F. McCabe, "Incorporation of antibacterial monomer MDPB into dentin primer," Journal of Dental Research, vol. 76, no. 3, pp. 768-772, 1997.

[22] N. Beyth, Y. Houri-Haddad, L. Baraness-Hadar, I. YudovinFarber, A. J. Domb, and E. I. Weiss, "Surface antimicrobial activity and biocompatibility of incorporated polyethylenimine nanoparticles," Biomaterials, vol. 29, no. 31, pp. 41574163, 2008.

[23] N. Beyth, I. Yudovin-Fearber, A. J. Domb, and E. I. Weiss, "Long-term antibacterial surface properties of composite resin incorporating polyethyleneimine nanoparticles," Quintessence International, vol. 41, no. 10, pp. 827-835, 2010.

[24] N. Beyth, A. J. Domb, and E. I. Weiss, "An in vitro quantitative antibacterial analysis of amalgam and composite resins," Journal of Dentistry, vol. 35, no. 3, pp. 201-206, 2007.

[25] M. M. Coogan and P. J. Creaven, "Antibacterial properties of eight dental cements," International Endodontic Journal, vol. 26, no. 6, pp. 355-361, 1993.

[26] E. J. DeSchepper, R. R. White, and W. von der Lehr, "Antibacterial effects of glass ionomers," American Journal of Dentistry, vol. 2, no. 2, pp. 51-56, 1989.

[27] W. Scherer, N. Lippman, and J. Kaim, "Antimicrobial properties of glass-ionomer cements and other restorative materials," Operative Dentistry, vol. 14, no. 2, pp. 77-81, 1989.

[28] E. Davidovich, E. Weiss, A. B. Fuks, and N. Beyth, "Surface antibacterial properties of glass ionomer cements used in atraumatic restorative treatment," Journal of the American Dental Association, vol. 138, no. 10, pp. 1347-1352, 2007.

[29] P. Karantakis, M. Helvatjoglou-Antoniades, S. TheodoridouPahini, and Y. Papadogiannis, "Fluoride release from three glass ionomers, a compomer, and a composite resin in water, artificial saliva, and lactic acid," Operative Dentistry, vol. 25, no. 1, pp. 20-25, 2000.

[30] Y. Torii, T. Itota, M. Okamoto, S. Nakabo, M. Nagamine, and K. Inoue, "Inhibition of artificial secondary caries in root by fluoride-releasing restorative materials," Operative Dentistry, vol. 26, no. 1, pp. 36-43, 2001. 

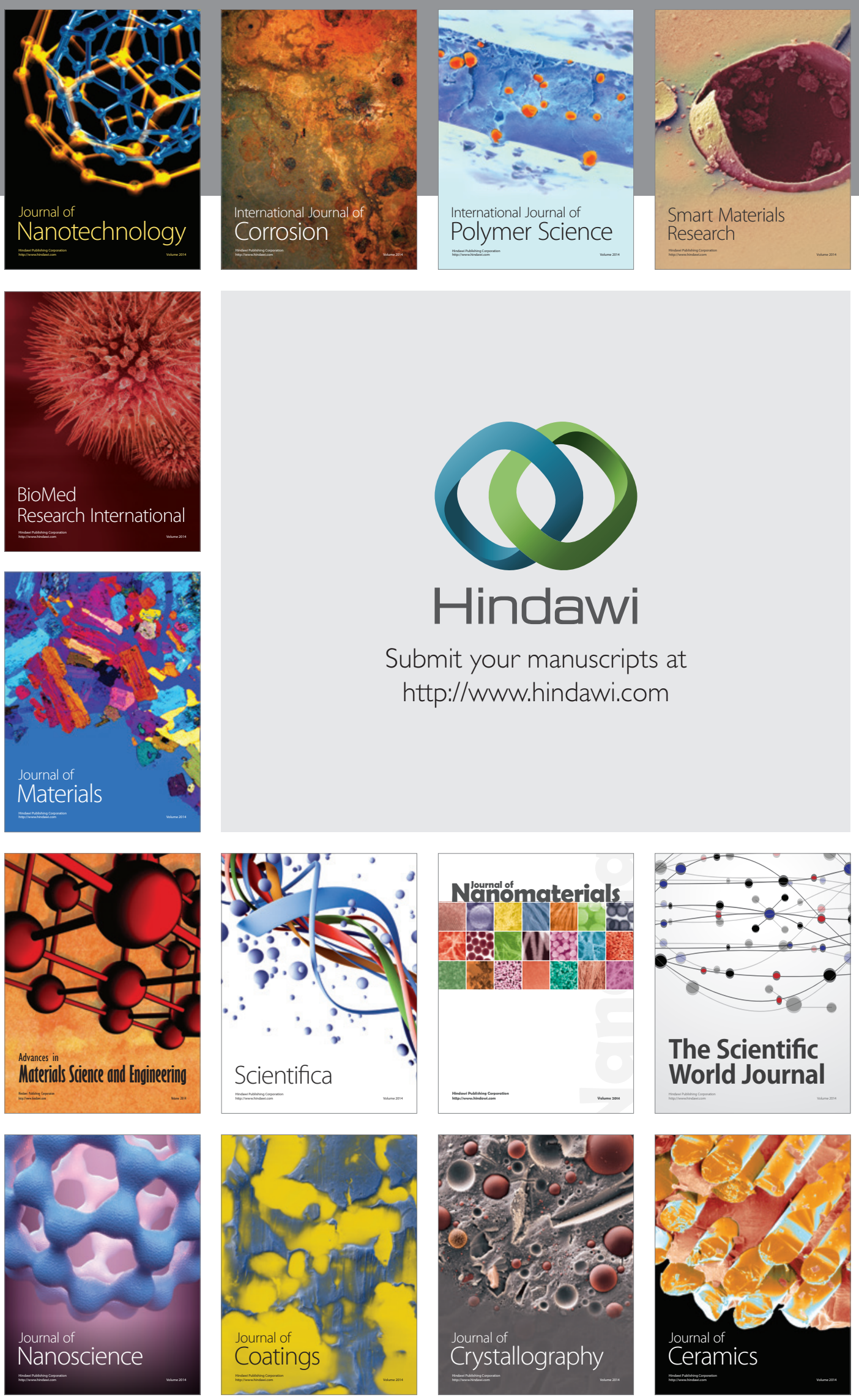

The Scientific World Journal

Submit your manuscripts at

http://www.hindawi.com

\section{World Journal}

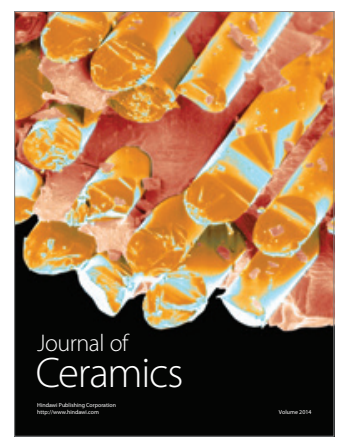

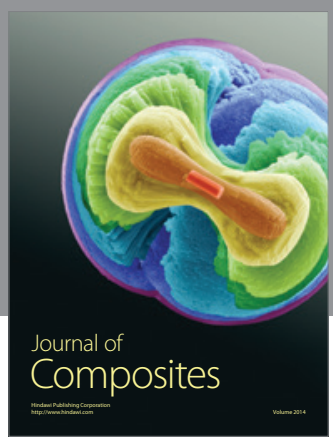
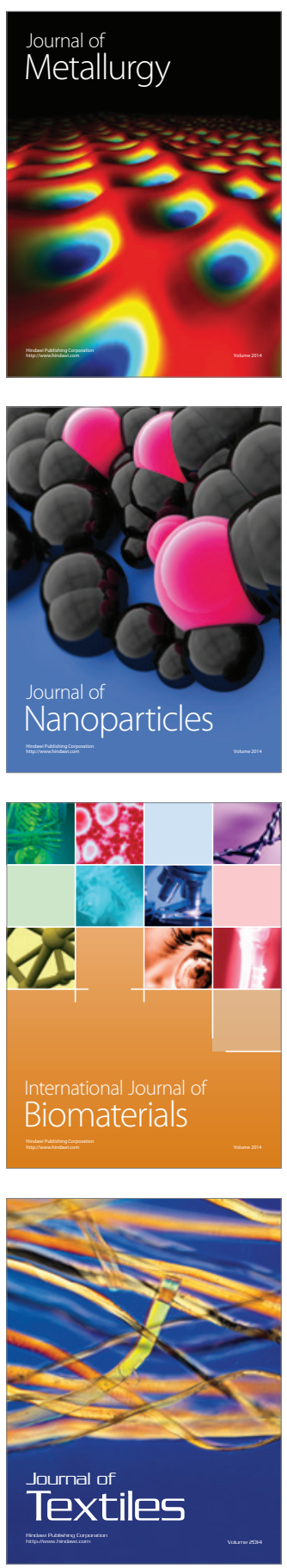